\section{Avaliação da qualidade das variáveis epidemiológicas e demográficas do Sistema de Informações sobre Nascidos Vivos, 2002}

\author{
Evaluation of quality of epidemiological and \\ demographic variables in the Live Births \\ Information System, 2002
}

\author{
1 Centro de Informação \\ Científica e Tecnológica \\ Fundação Oswaldo Cruz, Rio \\ de Janeiro, Brasil. \\ Correspondência \\ D. E. Romero \\ Departamento de \\ Informações em Saúde, \\ Centro de Informação \\ Científica e Tecnológica, \\ Fundação Oswaldo Cruz. \\ Av. Brasil 4365, Pavilhão \\ Haity Moussatché, sala 227, \\ Rio de Janeiro, $R J$ \\ 21045-900, Brasil. \\ dalia@cict.fiocruz.br
}

\begin{abstract}
This study evaluates the quality of data in the Brazilian Live Births Information System (SINASC), focusing on the methodological clarity of documentation and adequate data completeness and consistency at the national, regional, and State levels in 2002. The variables analyzed were: skin color/race of newborn, maternal schooling, maternal marital status, maternal occupation, maternal age, prenatal visits, live births, stillbirths, and birth weight. For most of the variables, SINASC shows good data completeness and consistency, but there were serious problems with the quality of data on previous children and maternal occupation. Related to race, there were some methodological problems in the definition and incomplete filling-in for the Federal District (Brasilia) and the States of São Paulo, Bahia, and Sergipe. Statistical analysis confirmed a significant association between data completeness and indicators of poverty and inequality. The study showed that improvements in SINASC data quality could make this system a good epidemiological source for identifying risk factors and socioeconomic conditioning factors.
\end{abstract}

Live Birth; Information Storage and Retrieval; Information Systems
Dalia E. Romero 1

Cynthia Braga da Cunha ${ }^{1}$

\section{Introdução}

É sobejamente notório, com base em revisão da literatura especializada, que a sobrevivência dos recém-nascidos, assim como suas condições de vida, está influenciada por uma diversidade de fatores, inter-relacionados entre si, como condições ambientais, sociais, genéticas e comportamentais, entre as quais se destacam os fatores maternos de reprodução (idade da mãe, paridade e intervalo intergenésico) 1,2 , as condições do nascimento (o peso ao nascer, a prematuridade $\mathrm{e}$ tipo de parto) e as condições sócio-econômicas (saneamento básico, educação materna, ocupação dos pais, estado marital materno) 3,4,5,6,7. Outros fatores reconhecidamente relevantes da saúde materno-infantil são a qualidade da atenção ao parto 8, o fumo 9,10,11,12 e as doenças maternas durante e prévias ao parto 13 .

Informações precisas, completas e oportunas de natureza epidemiológica representam insumos essenciais para o planejamento, monitoramento, execução e avaliação das ações de saúde, especialmente em países e regiões de ampla desigualdade sócio-econômica 14,15,16. No Brasil, a magnitude das desigualdades e os recursos escassos requerem que as prioridades para a gestão pública sejam embasadas em conhecimento sobre a natureza dessas desigualdades em saúde, assim como sobre sua distribuição espacial 17 .

Sendo assim, na área da saúde, a informação só ocupa espaço estratégico quando permite es- 
timar indicadores relevantes sobre a situação de saúde e fatores associados 18 , atende ao agente da decisão e reverte-se em ações concretas e resolutivas aos problemas de saúde no espaço onde são produzidas 19 .

Nesse sentido, em 2000, na $11^{a}$ Conferência Nacional de Saúde, reafirmou-se que a informação é um dos componentes principais para alcançar a eqüidade, qualidade e humanização dos serviços de saúde, fortalecer o controle social no âmbito do Sistema Único de Saúde (SUS), e melhorar a situação da saúde da população 20 . Em 2003, no documento A Construção da Política de Informação e Informática em Saúde do SUS 21 explicitou-se que as características sócio-demográficas, tais como idade, gênero, raça ou etnia, escolaridade, ocupação e classe social poderão ser utilizadas para a realização de estudos que objetivem a redução das desigualdades em saúde e a ampliação do acesso da população a serviços de qualidade, oportunos e humanizados. Em 2004, aprovou-se, pela Portaria no. 2.607/ GM 22, o "Plano Nacional de Saúde/PNS - um pacto pela saúde no Brasil”, no qual se reconheceu que o acompanhamento de algumas metas na área da saúde está limitado pela heterogeneidade das diferentes regiões e Estados brasileiros, pela qualidade e cobertura dos dados existentes nos sistemas de informação de base nacional, tais como o Sistema de Informações sobre Mortalidade (SIM) e o Sistema de Informações sobre Nascidos Vivos (SINASC).

O SINASC, implantado oficialmente a partir de 1990, desenvolvido à semelhança do SIM, não só tem o objetivo de contabilizar o número de nascimentos em todo território nacional, mas também de coletar dados epidemiológicos que permitam identificar as crianças que nascem com risco de vida, e intervir com maior rapidez para prevenir e até controlar os fatores de risco 23. Atualmente, o SINASC está implantado em todos os estados brasileiros e as informações são divulgadas pela página eletrônica do Departamento de Informação e Informática do SUS/Ministério da Saúde (DATASUS, http://www.datasus.gov. br), sendo disponíveis por município até o ano de 2002. O SINASC tem variáveis importantes, como: idade da mãe, sexo do recém-nascido, peso ao nascer, duração da gestação, grau de instrução da mãe, índice de Apgar, tipo de parto, paridade, estabelecimento onde ocorreu o parto e município de origem da mãe.

Já que a Declaração de Óbito (DO), documento do SIM, também apresenta a maioria dessas variáveis, poderiam ser obtidos coeficientes específicos de mortalidade infantil como insumos de análises mais minuciosas na área de saúde materno-infantil 24 , desde que ambos os siste- mas de informações apresentem boa cobertura dos eventos e que os dados obtidos tenham fidedignidade, de modo que as medidas estimadas representem os verdadeiros fatores de risco da população 25 .

O conhecimento da qualidade da informação do SINASC, tanto do ponto de vista qualitativo que implica a fidedignidade das informações, quanto quantitativo, que analisa a cobertura total dos eventos e a completitude do preenchimento, é fundamental para o conhecimento da potencialidade epidemiológica desse sistema $26,27,28$. Entretanto, escasseiam trabalhos que avaliem a qualidade da diversidade de variáveis do SINASC e que tenham abrangência nacional. $\mathrm{O}$ último divulgado, disponível só para as capitais brasileiras, refere-se a dados do período de 1992 a 1994 27, quando ainda não estava completamente implantado o SINASC em todo o território nacional.

Reconhece-se que, embora seja perceptível que a cobertura do SINASC esteja crescendo, ela ainda é deficiente em alguns Estados do país 29,30, sendo menor de $80 \%$ em Tocantins, Ceará, Paraíba, Pará e Alagoas no ano de 2002, segundo as estimativas da Rede Interagencial de Informações para a Saúde (RIPSA) 31. A cobertura é o aspecto mais contemplado em pesquisas recentes sobre qualidade da informação do SINASC, o que não será abordado no presente trabalho.

Neste trabalho, avaliou-se a qualidade da informação das variáveis do SINASC do ano 2002, último ano disponível, quanto à clareza metodológica de sua documentação, à completitude do preenchimento e à consistência dos resultados obtidos para todas as Unidades da Federação (UF) do Brasil.

\section{Material e método}

\section{Fonte de dados}

Os dados analisados correspondem às declarações de nascimento registradas no SINASC (3.059.402 nascimentos) no ano de 2002, último ano disponibilizado pelo Ministério da Saúde até a finalização desta pesquisa. O SINASC, através das declarações de nascimento, fornece informações sócio-demográficas, reprodutivas, assistenciais e de fatores de risco em aproximadamente vinte variáveis. Construiu-se no SPSS (SPSS Inc., Chicago, Estados Unidos) uma base de dados dos nascimentos.

O Censo Demográfico de 200032 constituiu a fonte de dados para o cálculo dos indicadores de referência com o objetivo de avaliar a consistência das informações do SINASC. Apesar da 
diferença de dois anos dessas bases de dados, considerou-se mais adequada essa comparação. Além da representatividade da amostra por UF, nas perguntas não incluídas no questionário do universo do Censo, é esperado que haja pouca ou nenhuma mudança no comportamento da fecundidade em um intervalo de tempo pequeno. Dos indicadores referentes às mães (idade ao parto, parturição, escolaridade, ocupação e estado civil), selecionaram-se no Censo as mulheres com um filho menor de um ano de idade. Para indicadores referentes aos nascimentos, como raça, selecionaram-se no Censo as crianças menores de um ano como uma aproximação dos nascimentos acontecidos no ano do levantamento da informação. Os dados do Censo foram processados no programa Redatam $+\mathrm{G} 4$ (Centro Latino-Americano e Caribenho de Demografia, Santiago, Chile).

\section{Variáveis selecionadas}

As variáveis sócio-econômicas disponíveis nas declarações de nascimento e avaliadas neste trabalho são: raça/cor da pele do recém-nascido, instrução materna, estado civil da mãe, ocupação materna e o número de consultas pré-natal. Informações a respeito do sexo do recém-nascido, história reprodutiva e da gestação (idade materna, total de filhos nascidos vivos e natimortos, peso ao nascer) também foram incluídos.

\section{Critérios de avaliação da qualidade}

\section{- Clareza metodológica}

A clareza metodológica refere-se às instruções de coleta, manuais de preenchimento e documentação da base de dados, assim como a comparabilidade das variáveis com outras fontes de informação quanto ao conceito e definição. Para isso, analisaram-se os manuais do SINASC: manual de procedimento 33 e manual de preenchimento 34 .

\section{- Incompletitude}

A incompletitude refere-se aos campos em branco (informação incompleta) de cada variável. Para a escolaridade materna, estado civil e consulta pré-natal, incluem-se os campos preenchidos com o código de informação ignorada, geralmente especificada no manual de preenchimento com o código 9. A inclusão desse código foi adotada, pois se demonstrou em outra pesquisa com dados do Sistema de Informação de Agravos de Notificação (SINAN) que a informação classificada como "informação ignorada" corresponde, em sua maioria, a campos sem informação e não à informação desconhecida por parte do informante 35 . Adotaram-se como ponto de referência para classificar a incompletitude os seguintes graus de avaliação: excelente (menor de $5 \%$ ), bom (5 a 10\%), regular (10 a 20\%), ruim (20 a $50 \%$ ) e muito ruim (50\% ou mais).

\section{- Consistência}

A consistência refere-se à plausibilidade dos resultados. Verificou-se se a informação declarada das variáveis, como recomendado por Thiru et al. 36 , apresentava coerência interna e se possuía valores considerados como aceitáveis quando comparados com as regularidades empíricas de alguns indicadores estimados com outra base de dados. A fonte externa utilizada na análise de consistência foi o Censo 2000. O cálculo dos indicadores de consistência foi realizado para todas as UF com incompletitude menor de $20 \%$ e sem a inclusão dos casos com informação ignorada, em branco ou com códigos errados. Os indicadores para avaliação da consistência variam segundo a disponibilidade de dados e de critérios pré-estabelecidos de qualidade da informação. As seguintes variáveis tiveram sua consistência analisada: a) Idade da mãe: utilizaram-se dois indicadores. O primeiro, o índice de Myers 37, consiste na quantificação da preferência de dígitos nas declarações da idade. Em seu cálculo, utilizamse idades simples, com a premissa de comportamento linear em sua variação; somam-se todos os efetivos da população de mulheres com idades que terminam nos dígitos de 0 até 9 - primeiro dos indivíduos de dez anos e mais de idade e, posteriormente, de vinte anos e mais. Essas duas séries se ponderam por fatores e se agregam uma com a outra para construir uma população fictícia, cuja distribuição deve ser uniforme quando não existe preferência por algum dígito (na situação de distribuição eqüitativa das idades, esperar-se-ia que em cada dígito a soma mencionada fosse $10 \%$ da soma total. Para não violar o pressuposto de linearidade, calculou-se o índice de Myers de mães de vinte anos ou mais de idade. Visando qualificar a intensidade da atração dos dígitos, utilizou-se a seguinte escala: baixo, de 0,0 a 5,0; mediano, de 5,1 a 15,0; alta, de 15,1 a 30,0; muito alta, para 30,1 e mais. O segundo indicador utilizado baseia-se na comparação entre o Censo 2000 e o SINASC, da idade média da mãe ao nascimento do filho. Para a estimativa desse indicador a partir do Censo 2000, selecionaramse as mulheres com filhos menores de um ano como aproximado dos nascimentos acontecidos no ano censitário.

b) Sexo: avaliou-se a qualidade da variável com a razão de sexo ao nascer, que é estimada por meio 
do quociente dos nascidos vivos do sexo masculino entre os nascidos vivos do sexo feminino no SINASC. Diversos estudos já mostraram estabilidade biológica próxima do valor de 1,06 na ausência de interferências sociais e contextuais, como abortos seletivos por sexo da criança e infanticídio feminino 38. Em países como o Brasil, sem indícios desse tipo de práticas, a razão de sexo é utilizada como medida de qualidade do registro e preenchimento da informação 39.

c) Peso ao nascer: o peso ao nascer foi avaliado através de dois indicadores. No primeiro, a razão de sexo da média do peso ao nascer, utilizou-se a constatação de Hindmarsh et al. ${ }^{40}$, segundo a qual o peso é maior para os recém-nascidos do sexo masculino. O segundo indicador é a classificação da qualidade dos dados de Battaglia \& Lubchenco ${ }^{41}$. Pela combinação da informação da duração da gestação com o peso ao nascer, gera-se a classificação em três categorias de qualidade da informação dos dados: errado, correto e provável erro. Para adequar o indicador a dados agrupados do peso ao nascer no SINASC, aplicou-se a adaptação realizada por Souza ${ }^{42}$. Entre algumas das especificações do método estão: se o recém-nascido possui um peso inferior a $500 \mathrm{~g}$, considera-se o dado errado; se localizado entre os percentis 10 e 90 da curva de Lubchenco, avalia-se como correto; se a duração da gestação é superior a 37 semanas com peso entre $1.500 \mathrm{e}$ 2.499g, considera-se como provável erro.

d) Total de filhos nascidos vivos: a parturição, mensurada como o número médio de filhos nascidos vivos que as mães tiveram até o momento do nascimento do último filho, é o indicador utilizado para avaliar a qualidade do número de filhos vivos declarados no SINASC, quando comparados com a obtida pelo Censo 2000.

e) Raça: estimou-se a diferença, entre o Censo 2000 e o SINASC, das porcentagens de nascidos declarados como brancos e como pardos.

f) Estado civil: estimou-se a diferença, entre o Censo 2000 e o SINASC, das porcentagens de mães que se declararam como casadas e como solteiras. Existem diferenças nas categorias dessas fontes de informação. Enquanto no Censo 2000 se pergunta sobre o estado civil (solteiro, casado, viúvo, divorciado e desquitado), no SINASC se indaga a situação conjugal (solteiro, casado, viúvo, separado judicialmente e união consensual). Para a comparação com o Censo 2000, juntaram-se as categorias solteiras e em união consensual desta última fonte de informação.

g) Escolaridade: estimou-se a diferença, entre o Censo 2000 e o SINASC, das porcentagens de mães que se declararam com nenhum grau de escolaridade e com 12 anos ou mais. h) Ocupação: estimou-se a diferença, entre o Censo 2000 e o SINASC, das porcentagens de mães que se declararam com alguma ocupação. Além disso, apresenta-se o percentual, do Censo 2000 e do SINASC, de mães ocupadas como empregadas domésticas e mães com alguma ocupação no setor agrícola.

\section{Análise estatística}

Calculou-se a correlação de Spearman, tendo como unidade de análise as UF, com o intuito de verificar se a incompletitude das variáveis do SINASC estava relacionada com a pobreza, a desigualdade econômica e de disponibilidade de recursos humanos em saúde. Selecionaramse diversos indicadores disponíveis por UF no Atlas de Desenvolvimento Humano 43. Como indicador de pobreza, usou-se o Índice de Desenvolvimento Humano (IDH) e, como medida do grau de concentração de renda, utilizou-se o índice de Gini, ambos indicadores do ano 2000. O número de médicos residentes por mil habitantes e o percentual de enfermeiros residentes com curso superior foram os indicadores selecionados sobre recursos humanos em saúde de cada UF

\section{Resultados}

\section{Clareza metodológica}

É confusa a instrução do manual quanto aos códigos que deveriam ser utilizados quando a informação é ignorada e quando não se aplica a pergunta. O manual possui a instrução: "Nenhum campo deve ser deixado em branco, colocando-se o código correspondente a Ignorado ou um traço (-), quando não se conhecer a informação solicitada ou não se aplicar ao item correspondente" 34 (p. 8, grifo nosso).

Embora exista clareza metodológica quanto ao preenchimento das variáveis sobre história reprodutiva das mulheres quanto à existência de filhos tidos anteriores, encontraram-se problemas em sua implementação. No manual de preenchimento, especifica-se que, quando não existirem filhos nascidos vivos ou natimortos, deve-se registrar $00 \mathrm{em}$ todas as caselas e, quando os dados forem ignorados, registrar 99. Entretanto, na base de dados existe um número elevado de declarações de nascimento com caselas em branco nessas variáveis (Tabela 1).

Em relação à raça do recém-nascido, não se especifica no manual quem deveria ser o informante desta variável. Sem esse conhecimento, fica a dúvida se a respondente é a mãe ou a pes- 
Percentual de incompletitude das variáveis do Sistema de Informações sobre Nascidos Vivos (SINASC) segundo Unidades da Federação (UF) e grandes regiões brasileiras, 2002

\begin{tabular}{|c|c|c|c|c|c|c|c|c|c|c|c|}
\hline UF & $\begin{array}{c}\text { Raça do } \\
\text { recém- } \\
\text { nascido }\end{array}$ & $\begin{array}{l}\text { Escola- } \\
\text { ridade } \\
\text { materna }\end{array}$ & $\begin{array}{c}\text { Estado } \\
\text { civil } \\
\text { materno }\end{array}$ & $\begin{array}{c}\text { Ocupação } \\
\text { materna }\end{array}$ & $\begin{array}{l}\text { Sexo do } \\
\text { recém- } \\
\text { nascido }\end{array}$ & $\begin{array}{c}\text { Idade } \\
\text { materna }\end{array}$ & $\begin{array}{c}\text { Filhos } \\
\text { nascidos } \\
\text { vivos }\end{array}$ & $\begin{array}{c}\text { Filhos } \\
\text { nascidos } \\
\text { mortos }\end{array}$ & $\begin{array}{c}\text { Peso ao } \\
\text { nascer }\end{array}$ & $\begin{array}{l}\text { Consultas } \\
\text { pré-natais }\end{array}$ & $\begin{array}{c}\text { Nascidos } \\
\text { vivos }\end{array}$ \\
\hline Norte & 0,76 & 2,19 & 0,95 & 2,78 & 0,12 & 0,12 & 17,38 & 39,75 & 1,16 & 1,57 & 301.208 \\
\hline Rondônia & 1,97 & 3,77 & 2,27 & 16,51 & 0,08 & 0,56 & 17,99 & 38,00 & 0,22 & 2,07 & 29.236 \\
\hline Acre & 2,85 & 2,80 & 0,88 & 3,50 & 0,02 & 0,08 & 23,11 & 35,84 & 5,89 & 2,72 & 15.841 \\
\hline Amazonas & 1,12 & 2,84 & 1,12 & 2,96 & 0,18 & 0,17 & 11,41 & 28,39 & 1,99 & 2,50 & 70.671 \\
\hline Roraima & 0,14 & 0,50 & 0,20 & 0,26 & 0,00 & 0,03 & 1,91 & 5,76 & 1,95 & 0,41 & 8.990 \\
\hline Pará & 0,21 & 1,79 & 0,78 & 0,03 & 0,13 & 0,00 & 21,95 & 49,52 & 0,53 & 1,11 & 136.000 \\
\hline Amapá & 0,21 & 2,25 & 0,89 & 2,60 & 0,26 & 0,09 & 24,79 & 76,66 & 0,23 & 1,63 & 14.196 \\
\hline Tocantins & 0,50 & 0,88 & 0,27 & 1,73 & 0,04 & 0,20 & 6,92 & 15,70 & 0,66 & 0,64 & 26.274 \\
\hline Nordeste & 13,02 & 5,69 & 8,21 & 11,47 & 0,26 & 0,50 & 17,04 & 32,48 & 1,45 & 3,32 & 929.717 \\
\hline Maranhão & 13,68 & 6,22 & 2,81 & 11,21 & 0,28 & 0,55 & 17,31 & 34,70 & 2,06 & 3,19 & 117.917 \\
\hline Piauí & 17,30 & 2,54 & 0,78 & 12,57 & 0,27 & 0,27 & 6,21 & 12,29 & 0,61 & 1,05 & 56.332 \\
\hline Ceará & 11,64 & 6,59 & 1,41 & 10,88 & 0,50 & 0,12 & 16,81 & 31,20 & 0,37 & 2,08 & 143.242 \\
\hline Rio Grande do Norte & 0,89 & 1,61 & 0,95 & 3,54 & 0,21 & 0,29 & 5,77 & 10,56 & 0,53 & 1,64 & 53.250 \\
\hline Paraíba & 8,61 & 6,86 & 2,29 & 10,25 & 0,23 & 0,31 & 13,38 & 25,05 & 0,65 & 3,26 & 64.006 \\
\hline Pernambuco & 2,51 & 2,08 & 1,75 & 5,40 & 0,07 & 0,11 & 3,94 & 7,49 & 0,53 & 1,10 & 156.053 \\
\hline Alagoas & 0,98 & 3,37 & 1,25 & 7,53 & 0,02 & 0,28 & 12,91 & 32,38 & 1,10 & 2,02 & 64.457 \\
\hline Sergipe & 43,87 & 6,40 & 2,14 & 10,21 & 0,01 & 0,35 & 28,13 & 62,24 & 0,91 & 4,60 & 37.085 \\
\hline Bahia & 21,77 & 9,14 & 27,06 & 19,06 & 0,33 & 1,21 & 31,15 & 55,67 & 3,22 & 6,66 & 237.375 \\
\hline Sudeste & 16,99 & 3,68 & 1,74 & 6,10 & 0,12 & 0,13 & 12,58 & 25,15 & 0,52 & 3,19 & 1.195 .168 \\
\hline Minas Gerais & 14,31 & 4,12 & 1,96 & 17,22 & 0,13 & 0,23 & 6,46 & 12,09 & 0,53 & 2,33 & 284.558 \\
\hline Espírito Santo & 3,66 & 1,41 & 0,57 & 4,71 & 0,17 & 0,16 & 7,01 & 14,09 & 0,30 & 1,25 & 55.076 \\
\hline Rio de Janeiro & 4,98 & 1,96 & 1,14 & 3,56 & 0,41 & 0,15 & 19,48 & 36,45 & 0,34 & 2,28 & 232.232 \\
\hline São Paulo & 23,87 & 4,33 & 1,97 & 2,09 & 0,00 & 0,08 & 13,30 & 27,89 & 0,60 & 4,10 & 623.302 \\
\hline Sul & 0,94 & 0,60 & 0,30 & 5,32 & 0,01 & 0,06 & 7,06 & 9,96 & 0,15 & 0,64 & 406.116 \\
\hline Paraná & 1,95 & 0,55 & 0,21 & 1,18 & 0,00 & 0,02 & 0,46 & 1,19 & 0,12 & 0,44 & 165.125 \\
\hline Santa Catarina & 0,35 & 0,78 & 0,41 & 12,03 & 0,04 & 0,07 & 2,09 & 6,04 & 0,10 & 0,95 & 85.730 \\
\hline Rio Grande do Sul & 0,19 & 0,57 & 0,34 & 6,03 & 0,01 & 0,10 & 16,83 & 21,47 & 0,21 & 0,69 & 155.261 \\
\hline Centro-oeste & 13,30 & 2,68 & 0,82 & 22,56 & 0,08 & 0,13 & 12,51 & 24,42 & 0,31 & 2,11 & 227.193 \\
\hline Mato Grosso do Sul & 0,42 & 2,25 & 0,48 & 6,66 & 0,01 & 0,07 & 5,14 & 10,66 & 0,68 & 1,23 & 39.933 \\
\hline Mato Grosso & 0,49 & 0,52 & 0,36 & 2,10 & 0,01 & 0,07 & 5,73 & 10,48 & 0,13 & 0,50 & 47.564 \\
\hline Goiás & 14,63 & 4,35 & 1,20 & 18,33 & 0,14 & 0,23 & 15,86 & 32,06 & 0,33 & 3,17 & 93.897 \\
\hline Distrito Federal & 35,09 & 1,90 & 0,82 & 66,36 & 0,07 & 0,03 & 19,13 & 35,25 & 0,11 & 2,39 & 45.799 \\
\hline Brasil & 11,78 & 3,66 & 3,37 & 8,52 & 0,14 & 0,24 & 13,67 & 26,75 & 0,80 & 2,65 & 3.059 .402 \\
\hline
\end{tabular}

Fonte: Cálculos próprios baseados no SINASC, 2002.

soa responsável pelo preenchimento (médico, enfermeiro, pessoal administrativo).

A ocupação materna apresenta diversos problemas de definição e codificação. Em primeiro lugar, o tempo de referência da ocupação não é definido como um intervalo de tempo determinado, como, por exemplo, a última ocupação nos últimos 12 meses. Pelo contrário, a instrução é vaga quando preceitua que seja registrada a ocupação exercida habitualmente pela mãe, sendo essa definida como o trabalho que a mãe vem desenvolvendo até o momento do parto ou a ocupação habitual anterior, no caso de mães aposentadas, para ser codificada posteriormente pelo órgão de processamento. Em segundo lugar, no manual não se especifica que a referência usada é a Classificação Brasileira de Ocupações (CBO), do Ministério do Trabalho e Emprego, de 1994 e não a de 2002, mais atualizada. 


\section{Incompletitude}

Em todas as UF do Brasil, observou-se excelente completitude de preenchimento nas variáveis idade materna, sexo do recém-nascido e estado civil da mãe (com exceção da Bahia), e de excelente a boa completitude nas variáveis escolaridade, número de consultas pré-natais e peso ao nascer (Tabela 1 e Figura 1).

Maior dispersão entre as UF encontrou-se na completitude do preenchimento da variável raça e ocupação. Dezoito UF têm menos de $10 \%$ de incompletitude da raça (todas as UF do Norte, Rio Grande do Norte, Paraíba, Pernambuco, Alagoas, Espírito Santo, Rio de Janeiro, todas as UF da Região Sul, Mato Grosso do Sul e Mato Grosso) enquanto outras UF são de completitude ruim (Distrito Federal, São Paulo, Bahia e Sergipe). Observou-se preenchimento muito ruim da variável ocupação no Distrito Federal e cerca de $20 \%$ de incompletitude em Minas Gerais, Bahia e Goiás.

As variáveis sobre história reprodutiva materna apresentaram os mais elevados percentuais de incompletitude em diversas UF. Entretanto, quatro UF (Roraima, Pernambuco, Paraná e Santa Catarina) possuem o preenchimento da parturição considerado como excelente e de bom a excelente para natimortos. As UF da Região Norte, com exceção de Roraima, apresentaram maior incompletitude dessas variáveis, seguidas das UF do Nordeste e do Sudeste. Em 15 UF a incompletitude da variável sobre natimortos é ruim ou muito ruim, principalmente nas UF de Sergipe, Amapá e Bahia.

\section{Consistência}

Mostrou-se a consistência da variável idade da mãe no SINASC, tanto pelo índice de Myers quanto pela proximidade com a idade média ao nascimento do filho estimada com os dados do Censo 2000 32. O índice de Myers indicou baixa intensidade da atração por determinados dígitos (o que reflete boa declaração da idade) em todas as UF das Regiões Sul, Sudeste, bem como no Distrito Federal, Mato Grosso do Sul, Tocantins, Sergipe, Acre, Ceará, Rio Grande do Norte e Pernambuco. No restante das UF, o índice de Myers classificou como de mediana atração, não superando em nenhuma UF o valor de 10. A diferença da idade média ao nascimento do filho entre o SINASC e o Censo 2000 é menor nas Regiões Sudeste, Centrooeste e Sul (Tabela 2). De acordo com o padrão esperado, pode afirmar-se que a variável sexo do recém-nascido é consistente, já que a razão de sexos é próxima a 1,06 na maioria das UF.

Constatou-se a consistência da variável peso ao nascer através dos resultados da razão de sexo da média do peso ao nascer e a classificação da qualidade dos dados de Battaglia \& Lubchenco 41 . $\mathrm{O}$ primeiro apresentou um peso maior para os recém-nascidos do sexo masculino, com grande homogeneidade entre os estados, variando de 1,03 a 1,04. O segundo indicador mostrou que os registros concentram-se na categoria "correto", indo de 93,67\% (Maranhão) a 96,13\% (Paraíba). No Maranhão e na Paraíba, encontraram-se os maiores percentuais de "provável erro": 6,16\% e $3,83 \%$, respectivamente.

A variável total de filhos nascidos vivos tem diferentes níveis de consistência segundo a UF. Pequenas diferenças entre a parturição média do Censo 2000 e do SINASC foram encontradas em Rondônia, Amazonas, Ceará, Minas Gerais, Espírito Santo, São Paulo, Santa Catarina, Rio Grande do Sul e Mato Grosso do Sul. Menor consistência foi encontrada no Distrito Federal e Rio de Janeiro, que pode ser conseqüência da expressiva incompletitude do preenchimento da variável sobre total de filhos nascidos vivos.

Quanto à variável raça/cor da pele da criança, observaram-se resultados do SINASC menos consistentes nas UF do Norte e na maioria das UF do Nordeste, já que foram acentuadas as diferenças, com o Censo 2000, das proporções de crianças de raça branca e parda. As diferenças maiores da proporção de nascimentos de brancos, entre o Censo 2000 e o SINASC, encontraram-se no Amapá, Acre, Tocantins e Ceará (Tabela 2). Por outro lado, notou-se que, no SINASC, declarouse com maior freqüência a cor parda da pele em detrimento da cor branca. Essas discrepâncias poderiam explicar-se por distintos informantes da pergunta, já que chamam a atenção para que as duas categorias comparadas estão, relativamente, compensadas entre as fontes. Em outras palavras, crianças que foram catalogadas pelo médico ou pessoal administrativo como pardas na declaração de nascimento, no Censo 2000 foram classificadas como brancas pelo declarante, este sendo normalmente a pessoa responsável do domicílio ou a mãe da criança.

A variável estado civil materno mostrou-se consistente (diferenças menores de $10 \%$ ) para todas as UF, com exceção do Piauí, onde o Censo 2000 registrou $18 \%$ a mais de mães casadas. Em geral, também se observou nesta variável que as diferenças compensam-se entre as categorias casada e solteira, o que poderia ser resultado das diferentes definições da variável em cada fonte de informação. Como mencionado na metodologia, o SINASC pergunta pela situação conjugal e no Censo 2000 a pergunta é sobre estado civil.

A variável escolaridade também apresentou 


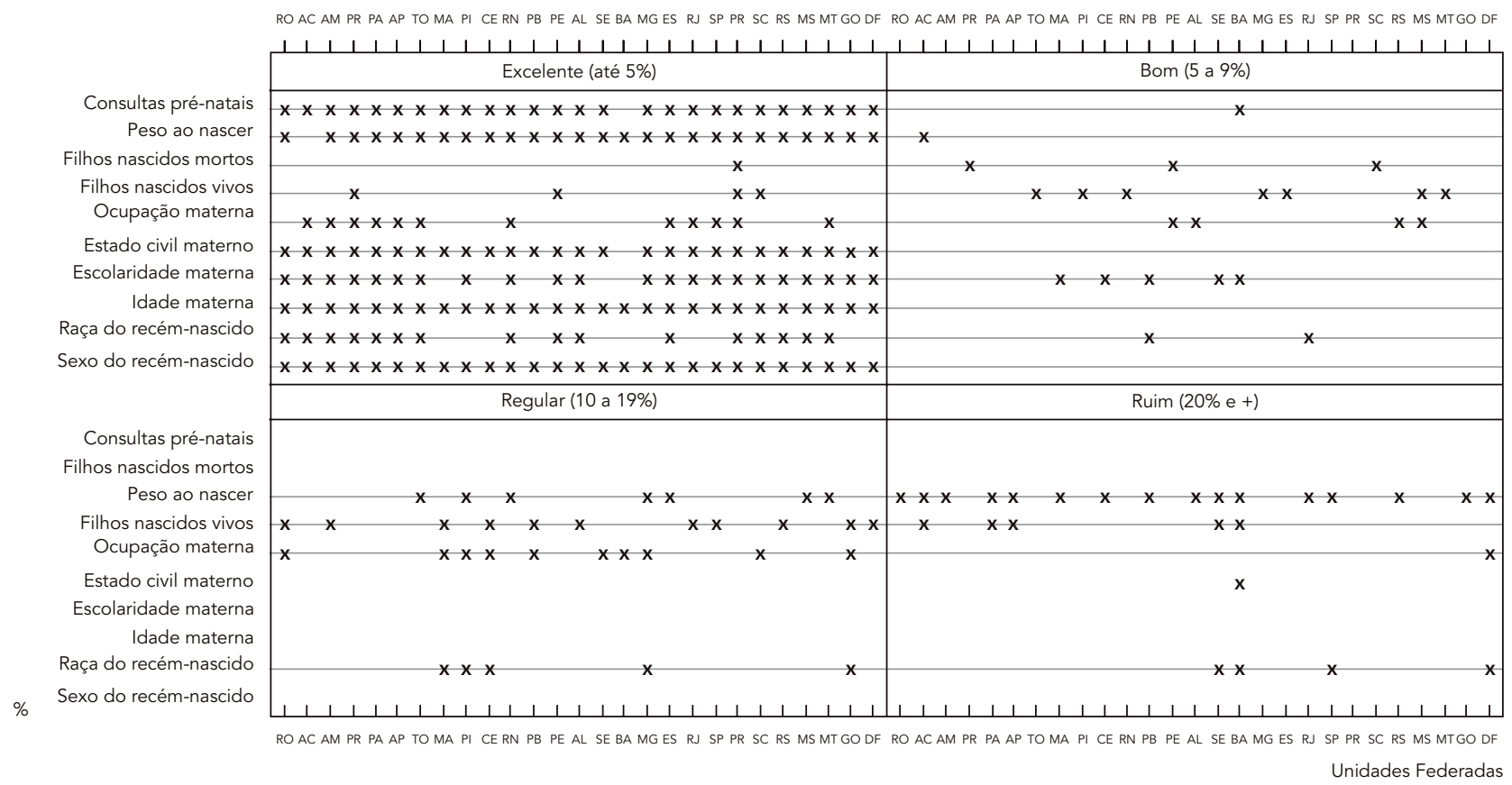

Fonte: Cálculos próprios baseados no SINASC, 2002.

resultados consistentes. Entretanto, observou-se que na categoria educacional de maior nível existem maiores diferenças entre as fontes comparadas. No Rio de Janeiro e em Goiás, o SINASC teve em torno de $10 \%$ a mais de mães com 12 anos e mais de escolaridade, ao passo que as diferenças são menores que $2 \%$ na categoria de nenhuma escolaridade. Assim sendo, pode afirmar-se que no SINASC se apresenta maior nível de escolaridade que no Censo 2000.

A variável ocupação não apresentou o mesmo nível de consistência que as variáveis anteriormente analisadas. Embora a proporção de mães ocupadas seja relativamente similar no Censo 2000 e no SINASC, observaram-se importantes diferenças quanto a categorias ocupacionais. Para exemplificar essas diferenças, a Tabela 3 apresenta as proporções de mães ocupadas em agricultura e como empregadas domésticas em cada uma das fontes de informação. Notou-se que no SINASC é menor a proporção das últimas e que a proporção em atividades agrícolas é substancialmente superior, quando comparadas com o Censo 2000. Segundo o SINASC, no Nordeste, $71 \%$ das mães estão em ocupação agrícola e, de acordo com o Censo 2000, essa proporção corresponde a $27 \%$. Assim, é cinco vezes superior a proporção de mães ocupadas como empregadas domésticas no Censo 2000.

\section{Correlação}

Na Tabela 4, apresentam-se os resultados dos coeficientes de correlação de Spearman, utilizado para avaliar a correlação entre a incompletitude do preenchimento da informação de diversas variáveis do SINASC, como a pobreza, a desigualdade econômica e a disponibilidade de recursos humanos em saúde. As variáveis peso ao nascer, escolaridade, idade, sexo e estado civil apresentaram correlação significativa da incompletitude com a desigualdade e o desenvolvimento das UF. A correlação positiva com o índice de Gini dessas variáveis é que a incompletitude das variáveis aumenta com a desigualdade das UF, especialmente para o peso ao nascer $\left(r_{\mathrm{s}}=0,64\right)$, idade $\left(\mathrm{r}_{\mathrm{s}}=0,57\right)$ e escolaridade $\left(r_{s}=0,46\right)$. Assim, a correlação negativa com o IDH revela que a UF com maior desenvolvimento apresenta o preenchimento mais completo das variáveis, sendo de maior intensi- 
Tabela 2

Indicadores de consistência das variáveis do Sistema de Informações sobre Nascidos Vivos (SINASC), idade materna, sexo do recém-nascido, peso ao nascer e filhos nascidos vivos, segundo Unidades da Federação (UF) e grandes regiões brasileiras, 2002.

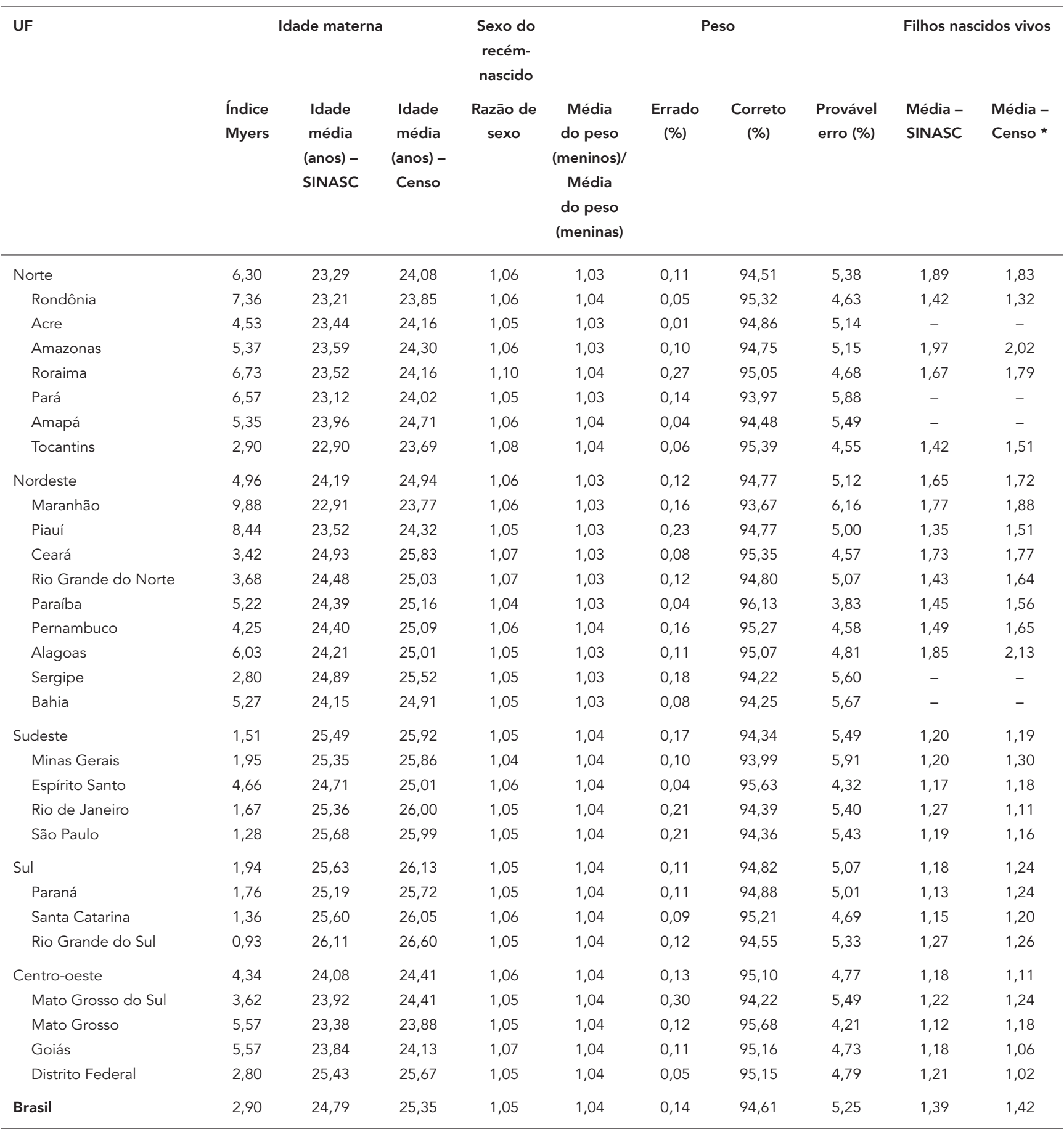

* Censo Demográfico de 200032.

Fonte: Cálculos próprios baseados no SINASC, 2002. 
Indicadores de consistência das variáveis do Sistema de Informações sobre Nascidos Vivos (SINASC): raça, estado civil, escolaridade e ocupação, segundo Unidades da Federação (UF) e grandes regiões brasileiras, 2002

\begin{tabular}{|c|c|c|c|c|c|c|c|c|c|c|c|c|}
\hline \multirow[t]{2}{*}{ UF } & \multicolumn{2}{|c|}{ Raça } & \multicolumn{2}{|c|}{ Estado civil } & \multicolumn{2}{|c|}{ Escolaridade } & \multicolumn{2}{|c|}{$\begin{array}{c}\text { Percentual de } \\
\text { ocupadas }\end{array}$} & \multicolumn{2}{|c|}{$\begin{array}{c}\text { Ocupadas como } \\
\text { empregadas } \\
\text { domésticas }\end{array}$} & \multicolumn{2}{|c|}{$\begin{array}{l}\text { Ocupadas na } \\
\text { agricultura }\end{array}$} \\
\hline & Branca & Parda & Casada & Solteira & Nenhuma & $\begin{array}{l}12 \text { anos } \\
\text { ou mais } \\
\text { anos }\end{array}$ & SINASC & Censo * & SINASC & Censo * & SINASC & Censo * \\
\hline Norte & 17,74 & $-18,54$ & $-0,92$ & 0,22 & 5,49 & $-5,46$ & 23,70 & 23,43 & 1,58 & 18,39 & 52,04 & 23,45 \\
\hline Rondônia & 9,46 & $-8,46$ & 3,45 & $-4,32$ & 2,16 & $-4,04$ & 25,73 & 25,09 & 1,27 & 18,13 & 65,59 & 19,05 \\
\hline Acre & 32,13 & $-34,68$ & 1,11 & $-2,25$ & 4,78 & $-4,04$ & 14,25 & 23,33 & 1,29 & 23,42 & 42,56 & 21,62 \\
\hline Amazonas & 15,42 & $-17,04$ & $-2,53$ & 1,94 & 8,50 & $-5,53$ & 27,08 & 23,71 & 2,08 & 16,46 & 62,73 & 32,84 \\
\hline Roraima & 10,38 & $-16,62$ & 0,12 & $-1,62$ & 3,91 & $-5,69$ & 21,51 & 30,50 & 2,13 & 18,45 & 12,23 & 8,95 \\
\hline Pará & 17,56 & $-17,77$ & $-0,52$ & $-0,10$ & 5,43 & $-5,70$ & 24,90 & 22,34 & 0,77 & 17,40 & 50,30 & 24,89 \\
\hline Amapá & 32,95 & $-36,17$ & $-8,89$ & 7,80 & 2,95 & $-0,78$ & 10,00 & 24,59 & 3,04 & 23,66 & 2,96 & 4,09 \\
\hline Tocantins & 20,43 & $-20,40$ & 0,29 & $-0,94$ & 3,56 & $-8,38$ & 20,14 & 24,17 & 4,94 & 23,96 & 40,51 & 10,93 \\
\hline Nordeste & 15,74 & $-15,62$ & $-4,11$ & 3,20 & 4,44 & $-5,52$ & 36,80 & 25,71 & 3,31 & 15,74 & 71,10 & 27,03 \\
\hline Maranhão & 12,94 & $-11,56$ & $-2,46$ & 2,09 & 5,49 & $-5,57$ & 43,20 & 27,01 & 2,01 & 14,34 & 84,24 & 43,00 \\
\hline Piauí & 12,45 & $-9,86$ & $-18,93$ & 18,44 & 4,43 & $-4,59$ & 33,94 & 26,31 & 7,84 & 12,48 & 77,27 & 34,80 \\
\hline Ceará & 25,97 & $-24,15$ & $-6,19$ & 4,93 & 3,96 & $-5,13$ & 37,59 & 26,49 & 1,86 & 16,36 & 69,26 & 16,50 \\
\hline Rio Grande do Norte & 14,34 & $-14,16$ & 1,65 & $-2,51$ & 2,92 & $-4,95$ & 31,82 & 22,59 & 5,91 & 17,79 & 52,59 & 12,70 \\
\hline Paraíba & 5,78 & $-3,93$ & 0,08 & $-1,37$ & 3,18 & $-3,95$ & 40,31 & 25,19 & 0,23 & 15,38 & 77,99 & 23,63 \\
\hline Pernambuco & 15,61 & $-14,87$ & 3,97 & $-4,97$ & 2,31 & $-5,05$ & 43,48 & 25,52 & 2,02 & 14,78 & 73,02 & 24,46 \\
\hline Alagoas & 20,50 & $-21,92$ & $-0,96$ & $-0,40$ & 3,62 & $-3,39$ & 25,18 & 24,23 & 2,83 & 17,04 & 64,33 & 34,94 \\
\hline Sergipe & - & - & 0,82 & $-2,26$ & 5,50 & $-7,48$ & 35,49 & 24,56 & 1,73 & 14,13 & 58,70 & 17,28 \\
\hline Bahia & - & - & - & - & 6,37 & $-6,95$ & 32,48 & 25,86 & 6,33 & 17,09 & 65,63 & 27,21 \\
\hline Sudeste & 0,33 & $-1,27$ & 5,86 & $-7,87$ & 1,76 & $-8,04$ & 28,34 & 29,61 & 5,78 & 18,69 & 3,69 & 5,48 \\
\hline Minas Gerais & 1,99 & $-2,43$ & 3,09 & $-4,65$ & 2,45 & $-6,88$ & 21,07 & 29,41 & 10,43 & 20,85 & 8,16 & 12,15 \\
\hline Espírito Santo & 7,63 & $-8,00$ & 5,08 & $-7,18$ & 2,07 & $-8,42$ & 29,56 & 31,74 & 6,50 & 19,73 & 31,72 & 18,92 \\
\hline Rio de Janeiro & 1,15 & $-2,96$ & 6,28 & $-7,84$ & 1,53 & $-10,01$ & 24,40 & 28,41 & 9,35 & 18,38 & 1,19 & 1,01 \\
\hline São Paulo & - & - & 6,81 & $-9,20$ & 1,43 & $-7,70$ & 32,49 & 29,99 & 3,57 & 17,64 & 1,07 & 2,53 \\
\hline Sul & $-6,20$ & 6,05 & 5,71 & $-7,64$ & 1,46 & $-7,84$ & 32,58 & 34,60 & 8,24 & 15,07 & 20,67 & 15,33 \\
\hline Paraná & $-10,48$ & 9,62 & 6,99 & $-8,79$ & 1,95 & $-8,68$ & 29,26 & 31,32 & 7,00 & 17,08 & 17,43 & 12,84 \\
\hline Santa Catarina & $-4,85$ & 4,55 & 6,92 & $-8,69$ & 1,12 & $-5,82$ & 36,56 & 36,84 & 5,96 & 12,47 & 22,31 & 18,81 \\
\hline Rio Grande do Sul & $-2,51$ & 3,26 & 3,95 & $-6,07$ & 1,18 & $-8,08$ & 34,24 & 36,69 & 10,68 & 14,75 & 22,86 & 15,58 \\
\hline Centro-oeste & 3,84 & $-4,11$ & 3,19 & $-4,57$ & 2,10 & $-8,22$ & 17,40 & 28,00 & 9,58 & 23,29 & 2,73 & 4,12 \\
\hline Mato Grosso do Sul & $-4,26$ & 2,53 & 2,38 & $-3,64$ & 2,96 & $-4,71$ & 16,55 & 26,18 & 8,59 & 25,85 & 2,42 & 7,26 \\
\hline Mato Grosso & 7,27 & $-9,30$ & 9,45 & $-10,82$ & 2,80 & $-7,90$ & 15,09 & 24,95 & 10,53 & 21,74 & 4,18 & 7,75 \\
\hline Goiás & $-0,17$ & 1,19 & 1,02 & $-2,30$ & 1,82 & $-10,63$ & 18,97 & 27,04 & 9,76 & 24,10 & 2,65 & 3,22 \\
\hline Distrito Federal & - & - & 1,65 & $-3,38$ & 1,02 & $-6,39$ & - & - & - & - & - & - \\
\hline Brasil & 6,04 & $-6,49$ & 1,83 & $-3,30$ & 3,03 & $-7,07$ & 30,24 & 28,34 & 5,05 & 17,55 & 34,26 & 14,58 \\
\hline
\end{tabular}

* Censo Demográfico de 200032.

Fonte: Cálculos próprios baseados no SINASC, 2002. 
Coeficientes de correlação entre a incompletitude das variáveis do Sistema de Informações sobre Nascidos Vivos (SINASC) e indicadores de pobreza, desigualdade econômica e disponibilidade de recursos humanos em saúde nas Unidades da Federação (UF), 2002.

\begin{tabular}{|c|c|c|c|c|}
\hline Variáveis & Índice de Gini & $\begin{array}{l}\text { Número de médicos } \\
\text { residentes por mil } \\
\text { habitantes }\end{array}$ & $\begin{array}{c}\text { Percentual de enfermeiros } \\
\text { residentes com } \\
\text { curso superior }\end{array}$ & $\begin{array}{c}\text { Índice de } \\
\text { Desenvolvimento } \\
\text { Humano do ano } 2000\end{array}$ \\
\hline Sexo & $0,381 *$ & $-0,184$ & $-0,081$ & $-0,384 \star \star$ \\
\hline Raça & 0,057 & 0,287 & $-0,020$ & $-0,167$ \\
\hline Idade & $0,575^{\star \star \star}$ & $-0,275$ & $-0,085$ & $-0,709 * \star \star$ \\
\hline Escolaridade & $0,458 * \star$ & $-0,172$ & $-0,105$ & $-0,512 * \star \star$ \\
\hline Estado civil & $0,363^{\star}$ & $-0,047$ & 0,081 & $-0,444 * \star$ \\
\hline Ocupação & 0,005 & 0,174 & 0,299 & $-0,117$ \\
\hline Filhos nascidos vivos (anterior) & 0,180 & $-0,186$ & 0,129 & $-0,260$ \\
\hline Filhos nascidos mortos (anterior) & 0,238 & $-0,248$ & 0,136 & $-0,288$ \\
\hline Peso & 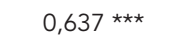 & $-0,395 * \star$ & $-0,200$ & $-0,651 * \star \star$ \\
\hline Consultas pré-natais & 0,229 & 0,054 & 0,001 & $-0,286$ \\
\hline
\end{tabular}

* $p$ valor $<0,10$;

** $\mathrm{p}$ valor $<0,05$;

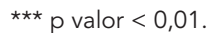

dade essa correlação nas variáveis mencionadas anteriormente $\left(r_{\mathrm{s}}=-0,65, \mathrm{r}_{\mathrm{s}}=-0,71\right.$ e $\mathrm{r}_{\mathrm{s}}=-0,51$, respectivamente). $\mathrm{O}$ sentido negativo da correlação da incompletitude com a disponibilidade de médicos nas UF, embora só seja significativa para o peso ao nascer $\left(r_{s}=-0,39\right)$, mostra que a menor disponibilidade desses recursos humanos influi na maior proporção de falta de preenchimento das variáveis. Contrariamente a esta constatação, nenhuma correlação foi observada com a disponibilidade nas UF de enfermeiros residentes com curso superior.

\section{Discussão}

Com relação aos eventos vitais, o conhecimento não apenas da totalidade, mas também das condições dos nascidos vivos, é imprescindível para fundamentar o planejamento de ações na área materno-infantil 26. O conjunto dos atributos dos nascimentos, como peso ao nascer e idade materna, e das condições sócio-econômicas da mãe, como escolaridade e ocupação, permitiria uma maior precisão na medição da desigualdade social e de riscos dos nascimentos. Carr-Hill 44 chama a atenção para a necessidade de considerar as variações sistemáticas dos indicadores de saúde a partir de uma adequada estratificação social. Szwarcwald et al. 45 , com base nos dados provenientes do SIM e do SINASC, mostraram os amplos gradientes, por escolaridade da mãe, das taxas de mortalidade neonatal e do baixo peso ao nascer

Os resultados do presente trabalho evidenciaram a potencialidade do SINASC como fonte de informação epidemiológica dos nascimentos. As declarações de nascimento, além de conterem informação relevante, sob a ótica individual, para a construção de indicadores preditores de nascimentos de risco, mortalidade infantil, problemas de desenvolvimento e riscos de doenças e agravos na infância, também dispõem de variáveis de endereço de residência e de ocorrência, o que, em princípio, possibilitaria a análise espacial do evento de interesse.

Neste estudo, mostrou-se que, para o ano analisado, o SINASC possui de boa a excelente completitude de preenchimento e consistência da informação na maioria das variáveis analisadas (idade materna, sexo do recém-nascido, estado civil da mãe, escolaridade e peso ao nascer). Contudo, observaram-se alguns problemas de qualidade da informação no SINASC. Assim como detectado por Mello-Jorge et al. 27 para os dados do SINASC de 1992 a 1994, encontrou-se uma completitude do preenchimento de ruim a regular, na maioria das UF, das variáveis sobre história reprodutiva materna: natimortos e filhos nascidos vivos anteriores. Portanto, as deficiências dessa informação impediriam o monitoramento da estreita associação, observada em diversos países e contextos, entre alta parturição e excesso de risco de morbidade e mortalidade infantil e materna 46,47 .

Os problemas observados na variável ocu- 
pação foram de diversas índoles: definição, codificação, completitude e consistência. Além do tempo de referência da ocupação não ser preciso, observaram-se sérias discrepâncias da informação com o Censo 2000, especialmente na categoria ocupacional "empregada doméstica" e "agricultura”. A CBO utilizada como referência é a de 1994, apesar da existência de uma classificação mais atual, de 2002. O uso desta facilitaria as comparações com outras fontes de informação, tais como o Censo 2000 e a Pesquisa Nacional por Amostra de Domicílio (PNAD). Logo, a variável ocupação mereceria ser estudada com maior aprofundamento.

Com a análise estatística, encontrou-se uma relação significativa entre a completitude do preenchimento e indicadores de pobreza e desigualdade. Em outras palavras, seria esperado que, em geral, UF com maior desenvolvimento tivesse melhor completitude. Apesar de a Bahia ser a UF que, sistematicamente, possui os maiores níveis de incompletitude em todas as variáveis analisadas, fato que confirmaria a correlação observada; chamam a atenção os altos níveis de incompletitude das variáveis ocupação e raça no Distrito Federal, assim como a incompletitude da última em São Paulo.

Essa variabilidade do grau de preenchimento, entre variáveis e entre UF, poderia estar associada ao interesse particular em determinadas variáveis, em detrimento de outras, por parte de gestores das Secretarias de Saúde. Carvalho ${ }^{48}$, com dados do SINASC de 1995, apontava que essa problemática assumiu proporções críticas no Estado de São Paulo, onde, apesar da boa cobertura do SINASC, não eram digitados os campos de diversas variáveis. Isso sugere a necessidade de implantação de atividades de capacitação para a melhora da coleta das variáveis do SINASC em quase todas as UF do país.

A indefinição do respondente da raça do recém-nascido é outro problema que deveria ser solucionado. Diversos estudos mostraram que, não somente a identificação da raça é subjetiva, mas que os critérios de mensuração têm um amplo efeito sobre os resultados de interesse que podem variar segundo contextos e observadores $49,50,51$.

Como assinalam Silva et al. ${ }^{52}$, deveria ser dispensada uma maior relevância à informação no contexto da atenção à saúde, aumentando as atividades de supervisão e controle de qualidade da coleta e processamento de dados do SINASC, redefinindo as variáveis com problemas metodológicos e incentivando os médicos e pessoal administrativo para o completo preenchimento de todas as variáveis contidas nas declarações de nascimento. Adicionalmente, é importante a resolução dos problemas que ocasionam o atraso na disponibilização da informação coletada pelo SINASC o qual continua sendo, como desde o começo de sua implantação, em torno de dois a três anos. Mello-Jorge et al. 27 e Carvalho 48, realizando as primeiras avaliações do SINASC, já apontavam para a necessidade de melhorar o fluxo entre a ocorrência, processamento, consolidação dos dados e disponibilização.

Algumas limitações deste estudo devem ser apontadas. Primeiramente, neste trabalho não foi considerado o efeito da cobertura da informação do sistema (relação quantitativa entre nascidos vivos informados no SINASC e estimados). Conhece-se que, apesar das melhoras observadas desde a implantação do SINASC, a cobertura ainda é deficiente em diversas áreas do país, embora de menor intensidade que as do SIM ${ }^{29}$. A RIPSA 31 estimou para 2002 uma cobertura menor que $80 \%$ para Ceará, Tocantins, Pará, Paraíba e Alagoas. Nesse sentido, se o aumento da cobertura dos nascimentos do SINASC não é acompanhado de melhorias na qualidade do preenchimento, nos próximos anos poderiam elevar-se os problemas de completitude e consistência da informação ao incorporar mais registros de nascimentos de áreas geográficas mais pobres.

Em segundo lugar, a seleção dos indicadores de avaliação da consistência foi restringida em função da indisponibilidade de outras fontes de informação mais recentes, como seriam as Pesquisas Nacionais sobre Demografia e Saúde da Mulher e da Criança (PNDS).

Outras reflexões quanto às limitações deste estudo referem-se aos indicadores escolhidos para a avaliação da consistência da informação. Por exemplo, a classificação de Battaglia \& Lubchenco 41 , utilizada para avaliar o peso ao nascer, pode ser suscetível a críticas já que depende da qualidade da variável idade gestacional. Porém, no Brasil não existem trabalhos recentes, com abrangência nacional, que avaliem esta última. Outra questão é de quanto seria o peso mínimo suficiente para que existam sinais de vida ao nascer, pois Battaglia \& Lubchenco ${ }^{41}$ consideram o peso inferior a $500 \mathrm{~g}$ como informação errada.

Espera-se, com este estudo, não somente oferecer subsídios para a melhora da qualidade das informações sobre os nascimentos no Brasil e estimular o uso do SINASC como sistema de monitoramento das condições de saúde materno-infantil em níveis desagregados como municípios, mas também propor alguns critérios de avaliação que poderiam ser estabelecidos sistematicamente na avaliação da qualidade da informação dos registros vitais. 


\section{Resumo}

O trabalho avalia a qualidade da informação do Sistema de Informações sobre Nascidos Vivos (SINASC) quanto à clareza metodológica da documentação, completitude do preenchimento e consistência para o país, grandes regiões e Unidades da Federação no ano 2002. Adicionalmente, correlaciona-se a completitude com indicadores de pobreza, desigualdade e recursos humanos. As variáveis analisadas são: raça/cor do recém-nascido, instrução materna, estado civil materno, ocupação materna, idade materna, consulta prénatal, filhos nascidos vivos, filhos nascidos mortos e peso do bebê ao nascer. Mostrou-se que o SINASC possui boa completitude de preenchimento e consistência da informação na maioria das variáveis; porém, observaram-se sérios problemas de qualidade nas variáveis sobre filhos anteriores e ocupação. Quanto à raça, encontraram-se tanto problemas metodológicos de definição da variável como incompletitude do preenchimento no Distrito Federal, São Paulo, Bahia e Sergipe, Brasil. Por intermédio da análise estatística, confirmou-se a relação significativa entre completitude e indicadores de pobreza e desigualdade. Espera-se que melhorias da qualidade da informação do SINASC possam contribuir para que esse sistema seja uma robusta fonte de dados epidemiológicos que permita identificar fatores de risco e condicionantes sócio-econômicos.

Nascido Vivo; Armazenamento e Recuperação da Informação; Sistemas de Informação

\section{Referências}

1. Naeye RL. Maternal age, obstetric complications, and the outcome of pregnancy. Obstet Gynecol 1983; 61:210-6

2. Palloni A. Assessing the effects of intermediate variables on birth interval-specific measures of fertility. Popul Index 1984; 50:623-57.

3. Meegama SA. Socio-economic determinants of infant and child mortality in Sri-Lanka: an analysis of post-war experience. Voorburg: International Statistical Institute; 1980. (Scientific Report, 8).

4. Victora CG, Barros FC. Infant mortality due to perinatal causes in Brazil: trends, regional patterns and possible interventions. São Paulo Med J 2001; 119:33-42.

5. Caldwell JC. Education as a factor in mortality decline an examination of Nigerian data. Popul Studies 1979; 33:395-413.

6. Hobcraft J, McDonald JW, Rutstein SO. Childspacing effects on infant and early child mortality. Popul Index 1983; 49:585-618.

\section{Colaboradores}

D. E. Romero e C. B. Cunha participaram da idealização, análise e revisão do artigo.

\section{Agradecimentos}

Este trabalho foi financiado pelo Centro de Informação Científica e Tecnológica, Fundação Oswaldo Cruz no âmbito do Projeto de Indução à Pesquisa e ao Desenvolvimento Tecnológico.
7. Cleland JG, van Ginneken JK. Maternal education and child survival in developing countries: the search for pathways of influence. Soc Sci Med 1988; 27:1357-68.

8. Wiggesworth JS. Monitoring perinatal mortality: a pathophysiological approach. Lancet 1980; 27:684-6.

9. Stein Z, Kline J. Smoking, alcohol and reproduction. Am J Public Health 1983; 73:1154-6.

10. Hofvendahl EA. Smoking in pregnancy as a risk factor for long-term mortality in the offspring. Paediatr Perinat Epidemiol 1995; 9:381-90.

11. Faden VB, Graubard BI, Dufour M. The relationship of drinking and birth outcome in a US national sample of expectant mothers. Paediatr Perinat Epidemiol 1997; 11:167-80.

12. Zambonato AM, Pinheiro RT, Horta BL, Tomasi E. Fatores de risco para nascimento de crianças pequenas para idade gestacional. Rev Saúde Pública 2004; 38:24-9. 
13. Carrapato MR, Marcelino F. The infant of the diabetic mother: the critical developmental windows. Early Pregnancy 2001; 5:57-8.

14. Krieger N, Chen JT, Waterman PD, Soobader MJ, Subramanian SV, Carson R. Choosing area based socioeconomic measures to monitor social inequalities in low birth weight and childhood lead poisoning: The Public Health Disparities Geocoding Project (US). J Epidemiol Community Health 2003; 57:186-99.

15. Winkler WE. Methods for evaluating and creating data quality. Information Systems 2004; 29:531-50.

16. Travassos C, Santos SM, Szwarcwald CL, Barcellos C, Romero D, Bastos FI, et al. Variáveis sociais nos bancos de dados de interesse para a área de saúde. In: I Seminário Nacional de Informação e Saúde. O Setor Saúde no contexto da Sociedade da Informação. Rio de Janeiro: Editora Fiocruz; 2000. p. 35-43.

17. Drachler ML, Côrtes SMV, Castro JD, Leite JCC. Proposta de metodologia para selecionar indicadores de desigualdade em saúde visando definir prioridades de políticas públicas no Brasil. Ciênc Saúde Coletiva 2003; 8:461-70.

18. Moraes IHS. Informações em saúde: da prática fragmentada ao exercício da cidadania. São Paulo: Editora Hucitec; 1994.

19. Paiva NST, Anselmi ML, Santos CB. Projeto "Viver em Cascavel”: análise do fluxo de informações. Rev Latinoam Enfermagem 2002; 10:537-43.

20. Ministério da Saúde. Política nacional de informação e informática em saúde - proposta, versão 2.0. http://politica.datasus.gov.br/PolíticaInformação Saúde\%202.0\%2029Março2004.doc (acessado em 29/Mar/2004).

21. Ministério da Saúde. A construção da Política de Informação e Informática em Saúde do SUS. http://politica.datasus.gov.br/PoliticaInforma caoSaude24_07_2003.pdf (acessado em 22/ Mar/2004)

22. Brasil. Portaria no. 2.607/GM de 10 de dezembro de 2004. Aprova o Plano Nacional de Saúde/PNS - um pacto pela saúde no Brasil. Diário Oficial da União 2004; $13 \mathrm{dez}$.

23. Mello-Jorge MHP, Gawryszewski VP, Lattorre MR. I - Análise dos dados de mortalidade. Rev Saúde Pública 1997; 31:5-25.

24. Szwarcwald CL, Bastos FI, Esteves MA, Andrade CL, Paez MS, Medici EV, et al. Desigualdade de renda e situação de saúde: o caso do Rio de Janeiro. Cad Saúde Pública 1999; 15:15-28.

25. Mello-Jorge MHP, Gotlieb SND, Sobol MLMS, Almeida MF, Lattorre MR. Avaliação do sistema de informação sobre nascidos vivos. Inf Demogr 1995; 29:1-4.

26. Mello-Jorge MHP, Gotlieb SND, Sobol MLMS, Almeida MF, Lattorre MR. Avaliação do sistema de informação sobre nascidos vivos e o uso de seus dados em epidemiologia e estatísticas de saúde. Rev Saúde Pública 1993; 27:1-46.

27. Mello-Jorge MHP, Gotlieb SND, Sobol MLMS, Almeida MF, Lattorre MR. O sistema de informação sobre nascidos vivos: primeira avaliação dos dados brasileiros. Inf Epidemiol SUS 1996; 2:15-48.
28. Silveira MH, Laurenti R. Os eventos vitais: aspectos de seus registros e inter-relações da legislação vigente com as estatísticas de saúde. Rev Saúde Pública 1973; 7:37-50.

29. Schramm JMA, Szwarcwald CL. Sistema hospitalar como fonte de informações para estimar a mortalidade neonatal e a natimortalidade. Rev Saúde Pública 2000;34:272-9.

30. Szwarcwald CL, Leal MC, Andrade CL, Souza Jr. PRB. Estimação da mortalidade infantil no Brasil: o que dizem as informações sobre óbitos e nascimentos do Ministério da Saúde? Cad Saúde Pública 2002; 18:1725-36.

31. Rede Interagencial de Informações para a Saúde/ Organização Pan-Americana da Saúde. Indicadores e dados básicos - Brasil, 2004/IDB-2004. Brasília: Ministério da Saúde; 2004.

32. Instituto Brasileiro de Geografia e Estatística. Censo demográfico de 2000. Rio de Janeiro: Instituto Brasileiro de Geografia e Estatística; 2002.

33. Ministério da Saúde. Manual de procedimento do Sistema de Informação sobre Nascidos Vivos. Brasília: Ministério da Saúde; 1999.

34. Ministério da Saúde. Manual de preenchimento do Sistema de Informação sobre Nascidos Vivos. Brasília: Ministério da Saúde; 1999.

35. Cruz MM, Toledo LM, Santos EM. O sistema de informação de AIDS do Município do Rio de Janeiro: suas limitações e potencialidades enquanto instrumento da vigilância epidemiológica. Cad Saúde Pública 2003; 19:81-9.

36. Thiru K, Hassey A, Sullivan F. Systematic review of scope and quality of electronic patient record data in primary care. BMJ 2003; 326:1070.

37. Myers RJ. Errors and bias in the reporting of ages in census data. Transactions of the Actuarial Society of America 1940; 41(Pt 2):395-415.

38. Bignami-Van Assche S. A different perspective on the imbalance of reported sex ratios at birth in rural China. Stanford Journal of East Asian Affairs 2004; 4:50-67.

39. Carvalho JAM, Sawyer D, Rodrigues RN. Introdução a alguns conceitos básicos e medidas demográficas. 2a Ed. Belo Horizonte: Associação Brasileira de Estudos Populacionais; 1994. (Série Textos Didáticos, 1).

40. Hindmarsh PC, Geary MPP, Rodeck CH, Kingdom JCP, Cole TJ. Intrauterine growth and its relationship to size and shape at birth. Pediatr Res 2002; 52:263-8

41. Battaglia FC, Lubchenco LO. A pratical classification of newborn infants by birth weight and gestational age. J Pediatr 1967; 71:159.

42. Souza LM. Avaliação do Sistema de Informação sobre Nascidos Vivos - SINASC, Minas Gerais e mesorregiões, 2000 [Dissertação de Mestrado]. Belo Horizonte: Centro de Desenvolvimento e Planejamento Regional, Universidade Federal de Minas Gerais; 2004. 
43. Programa das Nações Unidas para o Desenvolvimento/Instituto de Pesquisa Econômica Aplicada/Fundação João Pinheiro. Atlas do desenvolvimento humano no Brasil. Brasília: Programa das Nações Unidas para o Desenvolvimento/Instituto de Pesquisa Econômica Aplicada/Fundação João Pinheiro; 2003.

44. Carr-Hill R. Time trends in inequalities in health. J Biosoc Sci 1988; 20:265-73.

45. Szwarcwald CL, Bastos FI, Andrade CLT. Medidas de desigualdad en salud: la discusión de algunos aspectos metodológicos con una aplicación para la mortalidad neonatal en el Municipio de Rio de Janeiro, 2000. Cad Saúde Pública 2002; 18:959-70.

46. Bai J, Wong FWS, Bauman A, Mohsin M. Parity and pregnancy outcomes. Am J Obstet Gynecol 2002; 186:274-8.

47. Haaga JG. How are older maternal age and grand multiparity related to infant health? Malays J Reprod Health 1989; 7:147-58.
48. Carvalho DM. Grandes sistemas nacionais de informação em saúde: revisão e discussão da situação atual. Inf Epidemiol SUS 1997; 4:7-46.

49. Hahn RA, Mulinare J, Teutsch SM. Inconsistencies in coding of race and ethnicity between birth and death in US infants. A new look at infant mortality, 1983 through 1985. JAMA 1992; 267:259-63.

50. Boehmer U, Kressin NR, Berlowitz DR, Christiansen CL, Kazis LE, Jones JA. Self-reported vs administrative race/ethnicity data and study results. Am J Public Health 2002; 92:1471-2.

51. Lieberson S, Waters MC. The ethnic responses of whites: what causes their instability, simplification, and inconsistency? Soc Forces 1993; 72: 421-50.

52. Silva AA, Ribeiro VS, Borba Jr. AF, Coimbra LC, Silva RA. Avaliação da qualidade dos dados do Sistema de Informações sobre Nascidos Vivos em 1997 1998. Rev Saúde Pública 2001; 35:508-14.

Recebido em 17/Out/2005

Versão final reapresentada em 25/Jan/2006

Aprovado em 18/Jul/2006 